\title{
Reciprocity Scheme Approach to Free-Riding Evasion in Grid System
}

\author{
Ogundele, Lukman Adebayo \\ Obafemi Awolowo University, Ile Ife, Nigeria.
}

\begin{abstract}
The crucialrule of Grid systems is intentional resource sharing among individual Grid users.However; there is an intrinsicfriction between individual prudence and collective welfare that threatens the viability of these systems. Grid systems have emerged as a popular alternative to traditional client-server architectures for resourcesdistribution and sharing. Different academic studies have observed high levels of free-riding in various Grid systems, leading some to suggest the imminent collapse of these systems as viable resource sharing mechanism. This observable fact was due to hidden-action, hidden information as well as betrayer of co-operation and confidence among the selfish participating users with diverse malicious intentions. In Grid system, the supply and demand theory is symbiotic since the supplier of resources to other users within the system also demands for resources supplied by others. Generally, this paper investigated into the intersection of economics and computer science in the designing of Grid systems that consisting of rational participants with diverse and egocentric interests.This diversity behaviour usually led to free-riding which is a great hindrance to the achievement of the objectives of Grid system.This papertherefore develops an analytic model to analyze the behaviour of different users with different intentionsin Grid system. Reciprocity-based schemes that premised on incentive mechanisms and user'sco-operation was applied to address the problem of free-riding. In all, forty-five simulations were carried out under each parameter (i.e. storage space, memory and bandwidth). Before arriving at the result, various values of $\alpha$ used are; 0.05, 0.1, 0.5, 0.9, 1.0, 1.05, 1.1, 1.5 and 2.0 where $\alpha$ is the resource utilization determinant of the system. The proposed scheme performed optimally when $\alpha=1$. In the control experiments, it was observed that immediately the value of a is greater than 1, no matter how small, there exists contention and free-riding eventually ensue in the system. However, the value of $\alpha$ under the proposed scheme can never be greater than 1; since $0<\alpha \leq 1$. This circumstance is being controlled and managed by the formulated policy engine and access control mechanism of the scheme.
\end{abstract}

Keywords: Co-operation, Free-Riding,Grid System,Hidden-action, Hidden information, Incentives, and Reciprocity

\section{Introduction}

A Grid is a system that integrates and coordinates resources and users that live within different control domains and addresses the issues of security, policy, membership, and so on, that arise in these settings. Grid system allows its constituent resources to be used in a coordinated fashion to deliver various qualities of service, such as response time, throughput, availability, security, allocation and co-allocation of multiple resources to meet complex users' demands so that the utility of the combined system is significantly greater than that of the sum of its fragmented integral parts. As extracted from (Buyya 2002; Berman and Fox, 2003; Nabrzyski and Schopf 2003), any Grid system is supposed to be geographically distributed and heterogeneous. It must have large scale resource sharing and coordination capability with transparent, dependable, consistent and pervasive access. Grid system typically controlled its resources within self-interested local administrative domains and shared among competing users, Foster (2002). The above characteristics among others are very useful to determine the limits of the grid concept as well as to explore new application fields in grid computing. The above characteristics can be employed to determine the potential benefits a Grid infrastructure provides to collaborative resource sharing.

However, some systems do not qualify as Grids. For instance, a cluster management system such as Sun's Sun Grid Engine, Platform's Load Sharing Facility or Veridian's Portable Batch System when installed on a parallel computer or local area network can deliver and guarantees quality of service and thus constitute a powerful Grid resource. These systems are not Grid themselves, due to their centralized control of the hosts that they manage. They also have complete knowledge of system state, users' requests and complete control over individual components. Also, the Web is not (yet) a Grid since its open general-purpose protocols only support access to distributed resources but not the coordinated use of those resources to deliver interesting qualities of service, QoS.Generally, user behaviour can have potentially devastating effects on Grid systemperformance. Great strides have been made and valuable lessons have been drawn by the research community in the last few years. For example, researchers now appreciate the importance of identifying and tackling rational manipulation at all stages, including revelationof hidden-action, hidden information, betrayer of co-operation 
and confidence,and execution of tasks. At the same time, it is clear that further progress is needed on many fronts, e.g., insightson appropriate models and tools, so that the intellectual foundation is in place for the practice of economics-informed design of Grid systems.

\section{Grid Resources Allocation AND Management}

Grid resources are the physical or virtual components of limited availability within a system. Every device connected to a grid system as well as every internal system component is a resource. Virtual resources include files, network connections and memory. However, major resource types include; CPU time, Random Access Memory, Virtual Memory, Hard Disk space, Bandwidth and Network throughput. A resource handler is an identifier for a resource that is currently being accessed. Resource handler can be opaque, in which case they are often integer numbers, or they can be pointers that allow access to further information. Common resource handlers are file descriptors and sockets.

The resources that are coupled in grid computing environment are geographically distributed and different individuals or organizations own each one of them and have their own access policies, cost, and mechanisms. The resource owners manage and control resources using their favourite resource management and scheduling system (called local scheduler) and the Grid users are expected to honour that and make sure they do not interfere with resource owners' policies. They may charge different prices for different users for their resource usage and it may vary from time to time. The global resource management and scheduling systems, for example Nimrod/G, popularly called Grid schedulers or meta-schedulers, coordinate the user access to remote resources in cooperation with local schedulers e.g., Condor, Codine/GRD and LSF via grid middleware services, Abramson et al. (2000).

Traditionally, most of the schedulers follow system centric approach (for instance, they just care about system performance) in resource selection and often (completely) ignore the user requirements (for example, resource access cost). In order to overcome this problem, effective and efficient grid resource management and scheduling system architecture must be put in place. There is no single perfect solution that meets all users' requirements; hence the requirements (schedulers) are tailored for each class of applications. The key components of resourcemanagement system as observed by Buyya et al. (2000) are; Grid resource broker, job control agency, schedule advisor, Grid explorer, Grid manager, deployment agent and local resource manager.

\section{Previous Related Works}

As a generalization of the tit-for-tat scheme, Chu et al., 2004 propose a taxation scheme for Grid streaming applications. In accounting for the heterogeneity in upstream bandwidths among users, a tax schedule" specifies the amount of subsidy between resource rich and resource poor nodes. Many indirectreciprocity schemes have been proposed and they are often called reputation-based schemes. They differ from one another primarily in the computation of reputation scores and the mapping of scores to strategies (e.g., Dellarocas and Resnick, 2003; Gupta et al., 2003; Jurca and Faltings, 2005; Kamvar et al., 2003; Yu and Singh, 2000). Indirect-reciprocity schemes are more scalable than direct-reciprocity schemes, especially for Grid systems with large population sizes, highly dynamic memberships, and infrequent repeat transactions, Feldman et al., 2004a. Several studies propose statistical methods to detect misbehaviour and mitigate attacks on reputation systems. Buchegger and LeBoudec, 2004; propose a Bayesian approach, in which users monitor their neighbours' behaviour and periodically exchange this information. Users update their view based on their neighbors' reports if the information passes a Bayesian deviation test. This scheme redirects a more general approach calling for the storage of two reputation scores for each user: one that indicates the user's contribution level in the system, and a second one that reflects upon the user's trustworthiness in reporting information about other users.

Dellarocas, 2000, takes a different approach and proposes to alleviate these attacksthrough methods that are based on anonymity. Yet other researchers propose to base the computation of the reputation scores on the weighted graph that represents the interactions between the entities. This approach was often used to analyze false praise attacks. Kamvar et al., 2003, devise the EigenTrust algorithm that calculates the PageRank on the recommendation graph, where recommendations from trusted nodes are worth more. Feldman et al., 2004a, propose to apply the maxflow algorithm on the graph. The maxflow is the greatest level of reputation the source can give to the sink without violating "reputation capacity" constraints. This method alleviates collusion behaviour in which a group of non-cooperative users dishonestly report high reputation values on each other to subvert the reputation system. The effect of collusion is magnified in systems with cheap pseudonyms.

Incentives for Cooperation in Peer-to-Peer Systems can engage in the Sybil attack Douceur, 2002, that is, create fake identities and collude with their own multiple identities. Cheng and Friedman, 2005, formalize the notion of sybilproofness in reputation schemes. They demonstrate that if reputation is determined only by the structure of the graph and the edge values, then no Sybil-proof mechanism exists. Conversely, if reputation values are computed with respect to a fixed node in the graph, sybilproofness is guaranteed when several conditions are 
satisfied. In particular, some flow-based algorithms (similar to the algorithm presented in Feldman et al., 2004a satisfy the conditions of sybilproofness. Ayodeji et al. 2011, proposed autonomous hybrid resource management algorithm aim at optimizing the resource utilization of resources providers using "what-you-give-is-what-youget" Service Level Agreements resource allocation policy. Iyanda et al., 2011 also developed a prototype for the contention control in collaborativeresource sharing envinronment based on Token-exchange (TE) incentive.In this paper, reciprocity-based schemes that premised on incentive mechanisms and user's cooperation was applied to address the problem of free-riding. This is the major different from this paper and all other related works.

\section{The Proposed Reciprocity-Based Scheme}

A growing recognition of the potential that lies in reciprocity-based schemes hasled to an extensive amount of research in this area. Conversely, thereare some main issues that have not been explored. Such issues include hownewcomers should be treated in reciprocity-based schemes. The assumption of persistent identities in systems where identities are cheap and can be obtained or replaced at almost no cost is desirable for network growth as it encourages newcomers to join the system. However, this also allows misbehaving users to escape the consequences of their actions by repeatedly switching to new identitieswhile being indistinguishable from legitimate newcomers. There are two ways to counter this attack. The first is to require the use of free but irreplaceable pseudonyms, e.g., through the assignment of strong identities by a trusted central authority (Castro et al., 2002). In the absence of such mechanisms, it may be necessary to impose a penalty on all newcomers, including both legitimate newcomers and fraudsters which exacts a social cost (Friedman and Resnick, 1998; Feldman et al., 2004b; Feldman and Chuang, 2005). In reciprocity-based schemes,users maintain histories of past behaviour of other usersand use this information in their decision making processes where user $X$ decides how to serve user $Y$ based solely on the service that $Y$ has providedto $X$ in the past.

Reciprocity schemes are appropriate for applications with long session durations,as they provide ample opportunities for reciprocation between pairs ofusers. In this scheme, it is also possible to adopt a stranger policy that adapts to the observed behaviour of previous strangers, thereby avoiding unnecessary incurrence of the social cost. Systems that are based on reciprocity are not vulnerable to collusive behaviour such as false accusation and false praise since every user owns its private/public key through which it participates in the system. Identification, authentication, confirmation and authorization are done using unique and static ID mapping for access control of the system. For effective authorization, two distinct actions must be performed. Firstly authorization request must be decided upon and the result of the decision must be enforced. These two steps do not necessarily have to be carried out by the same entity but can be distributed between Policy Decision Point (PDP)and Policy Enforcement Points (PEP). The PDP is a logical entity that makes authorization decisions. As described in Farrell (2000), before making an authorization decision, three kinds of information are required. These are:the resource to be accessed and the desired method of accesse.g. read orwrite to a file; information about the requester, e.g. user name and/or group memberships and the operating environment, i.e. authorization information. It is possible that more than one rule from the policy applies to a requested access by a specific requester. A PEP is the logical entity that enforces a decision made by a PDP, that is, it technically realizes the grant or denial of a request. However, PEP does not make its own authorization decisions. The Policy Management Tool (PMT) is administrator dependent and responsible for determining which resources can be delegated and under what conditions (policies) delegation is permitted as a result of a collaborative arrangement. It is reasonable to implement the Policy Decision Point (PDP) in a way that the User Based Policy (UBP)is evaluated and enforced under the access control of the system. If access is already denied by the UBP in the access control of the system, the request does not have to be transmitted to and evaluated by the System Based Policy (SBP) at all.Using the assumption that the Utilization Rate, $\mathrm{Re}_{\mathrm{i}}$, of individual user is a function of both the incentive (I) and co-operation (C) scores, therefore, $R e_{i}=f\left(I_{i}, C_{i}\right)$. This was computed as the product of the incentive and co-operation scores. If both the incentive and co-operation scores increase the Utilization Rate therefore increases and vice versa, hence;

$$
\begin{aligned}
& \operatorname{Re}_{i}=f\left(\mathrm{I}_{\mathrm{i}}, \mathrm{C}_{i}\right)=I_{i} \times C_{i} \\
& \text { But } I_{i}=\frac{\boldsymbol{R}_{i}}{\mathrm{R}_{\text {tot }}} \\
& \text { and } C_{i}=\frac{\partial f}{\partial I_{i}} I_{i}+\frac{\partial f}{\partial R_{i}} R_{i}
\end{aligned}
$$


$\therefore \operatorname{Re}_{i}=\left(\frac{R_{i}}{R_{t o t}}\right) \times\left(\frac{\partial f}{\partial I_{i}} I_{i}+\frac{\partial f}{\partial R_{i}} R_{i}\right)$

The estimated optimal resource, $\mathrm{R}_{\mathrm{c}}$, to be consumed by each user is therefore incentive $(I)$ and co-operation (C) scores and resource contribution $\left(R_{i}\right)$ dependent, thus, $R_{c}=f\left(I_{i}, \mathrm{C}_{i}, \mathrm{Re}_{i}\right)=\alpha R_{i}$ (where $R_{c}$ is the estimated optimal resource to be consumed by the concerned user, $\alpha$ is the utility value called resource utilization determinant and $R_{i}$ is the resource contributed to the system by the affected user. To determine the value of $\alpha$, it was expressed in terms of utilization score. The total utilization of all the participating organizations was therefore computed as follows:

Total $(\mathbf{R e})=\mathrm{Re}_{1}+\mathrm{Re}_{2}+\mathrm{Re}_{3}+\mathrm{Re}_{4}+\mathrm{Re}_{5}+\ldots \ldots \ldots \ldots+\mathrm{Re}_{\mathrm{n}}$

$\therefore \operatorname{Total}(\mathrm{Re})=\sum_{i=1}^{n} \mathrm{Re}_{i}$

The utility value, $\alpha$, was estimated as the ratio of the utilization score of each organization to the total utilization scores of all the participating users.

$$
\therefore \alpha=\frac{\operatorname{Re}_{i}}{\sum_{i=1}^{n} \operatorname{Re}_{i}}
$$

Therefore; $0<\alpha \leq 1$

Since $\operatorname{Re}_{i}=\left(\frac{R_{i}}{R_{\text {tot }}}\right) \times\left(\frac{\partial f}{\partial I_{i}} I_{i}+\frac{\partial f}{\partial R_{i}} R_{i}\right)$, thereforefrom (2), it follows that the estimated optimal resource utilization of each user is incentive and co-operation scores as well as resource contribution dependent as required.

With the proposition that there exists pure strategy equilibrium such that out of all $n$ original users, $k(0 \leq k \leq n)$ users decide to share their resources while the rest (free-riders) do not.Also with the assumption that there are $k$ 1 users sharing resources, all original users will share resources if and only if:

$$
f\left(\frac{n}{k}\right) \mathrm{v}_{i}-c_{i} \geq f\left(\frac{n}{k-1}\right) v_{i}
$$

where $c i$ is the lump-sum cost of user $i$ and each user gets value $v i$ from each resource donated to the system, $c i$ and $v i$ are privately known to individual user. Also $f\left(\frac{n}{k}\right)$ is the probability that a user can use any desired resources from the system, $n$ is the total number of original users, and $k$ is the number of sharers.

Thatis;

$$
\frac{v_{i}}{c i} \geq \frac{1}{f\left(\frac{n}{k}\right)-f\left(\frac{n}{k-1}\right)}
$$

As long as (5) is satisfied, some original users are willing to share.

For the stability of equilibrium, let $i \in\{S H\}$ be the original set of sharers and $j \in\{N S\}$ be the set of nonsharers (free-riders). To show stability of the system, we need to show that $i \in\{S H\}$, and $j \in\{N S\}$ won't deviate unilaterally. For $i \in\{S H\}$, itrequires that:

$$
\frac{v_{i}}{c i} \geq \frac{1}{f\left(\frac{n}{k}\right)-f\left(\frac{n}{k-1}\right)}
$$

For $j \in\{N S\}$, it also requires that: 


$$
\frac{v_{i}}{c i} \leq \frac{1}{f\left(\frac{n}{k}\right)-f\left(\frac{n}{k-1}\right)}
$$

As long as (6) and (7) are satisfied, the equilibrium is stable given the network size $n$.

The above proposition therefore implies that there exist some users who are willing to share resources, thus, the system does not collapse. There are two possible reasons. First, in equilibrium, they are better off than nonsharing. Second, when they decide to share their resources, they believe that some other users might make the same decision. It is important to note that there are multiple pure strategy equilibria in this fixture. However, it may be difficult to ascertain beforehand which $k$ users might decide to share resources. But over time, the users will establish a routine where they will share resources over a period of time without free-riding. The architecture of User and System Based policy and Reciprocity-based scheme policy engine for the proposed system are depicting in Figures 1 and 2. The configuration of resources and access control of the scheme is presented in Figure 3

Process Engine

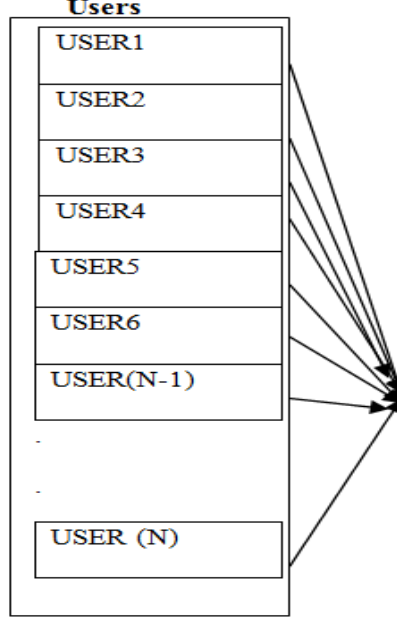

Figure1: Architecture of the proposed User and System Based policy for reciprocity-based scheme

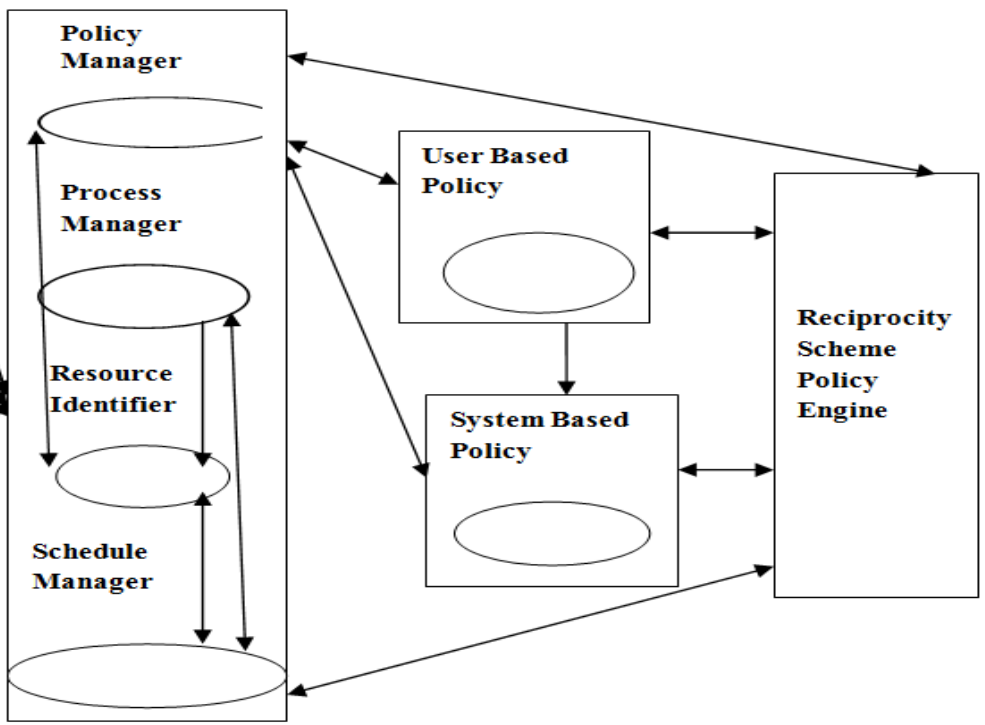

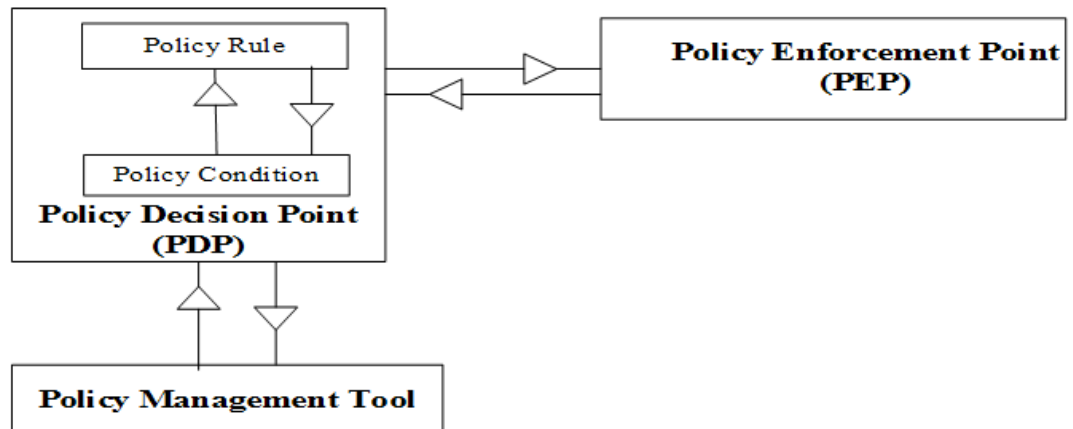

Figure 2: Extended Reciprocity-based scheme policy engine (Inspired from Westerinen, et al., 2001) 


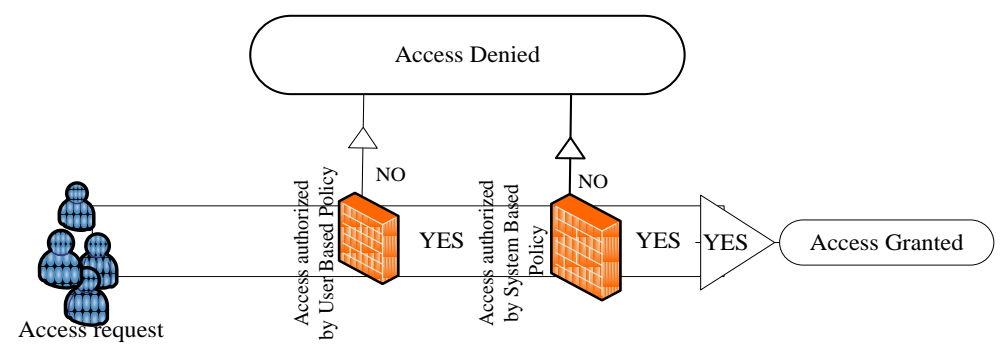

Figure 3: Resource and Access Control of the Reciprocity-based Scheme

\section{Experimental Results Discussion}

This section discussed the simulation results of the proposed scheme.The three resources considered in the simulation set up, are storage space, memory and bandwidth. The utilization of each resource was simulated under different scenarios using the condition $R_{c}=\alpha R_{\mathrm{i}}$ where $\mathrm{R}_{\mathrm{c}}$ being the resource contributed, $\mathrm{R}_{\mathrm{i}}$ being the estimated resource utilization and $\alpha$ is the resource utilization determinant. During simulation different values of $\alpha$ (resource utilization determinant) used for simulation were; $0.05,0.1,0.5,0.9,1.0,1.05,1.1,1.5$ and 2.0. Figures 4,5 and 6 as shown below depict the simulation results. With different values of $\alpha$, it was observed from the simulation results that the scheme perform well with all values of $\alpha$ less than or equals to 1 . For each value of $\alpha$, five simulations were carried out and the mean value of the output for each $\alpha$ was measured. In all, fortyfive simulations were carried out under each parameter (i.e. storage space, memory and bandwidth) before arriving at the result. Specifically, the proposed scheme performed optimally when $\alpha=1$. In the control experiment, it was observed that immediately the value of $\alpha$ is greater than 1 , no matter how small, there exists contention and free-riding eventually ensue in the system. However, the value of $\alpha$ under this proposed scheme can never be greater than 1 ; since the condition that $0<\alpha \leq 1$ is basic tool of this scheme. Thissituation is being controlled and managed by the formulated policy engine and access control mechanism of the scheme.

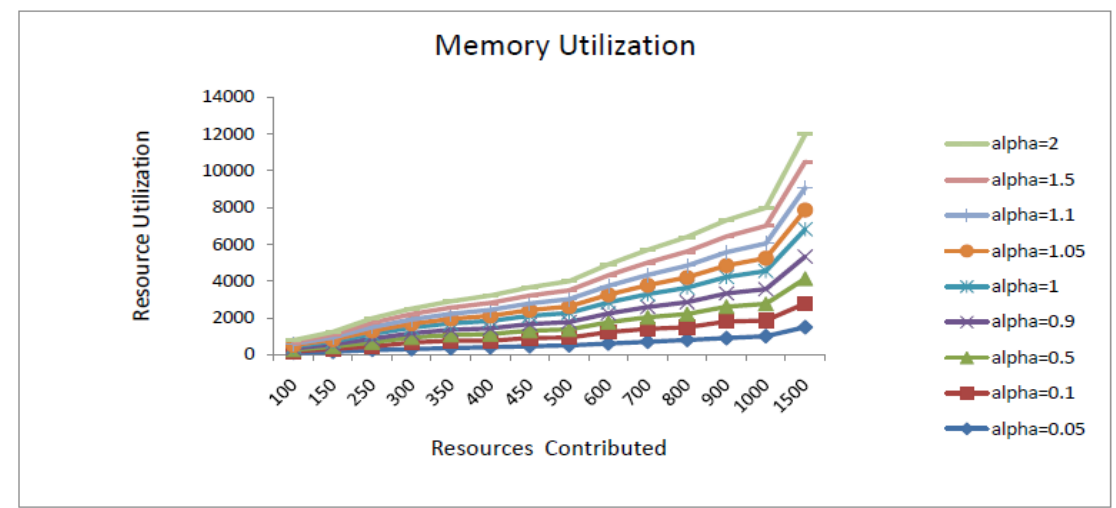

Figure 4: Simulation result of memory utilization

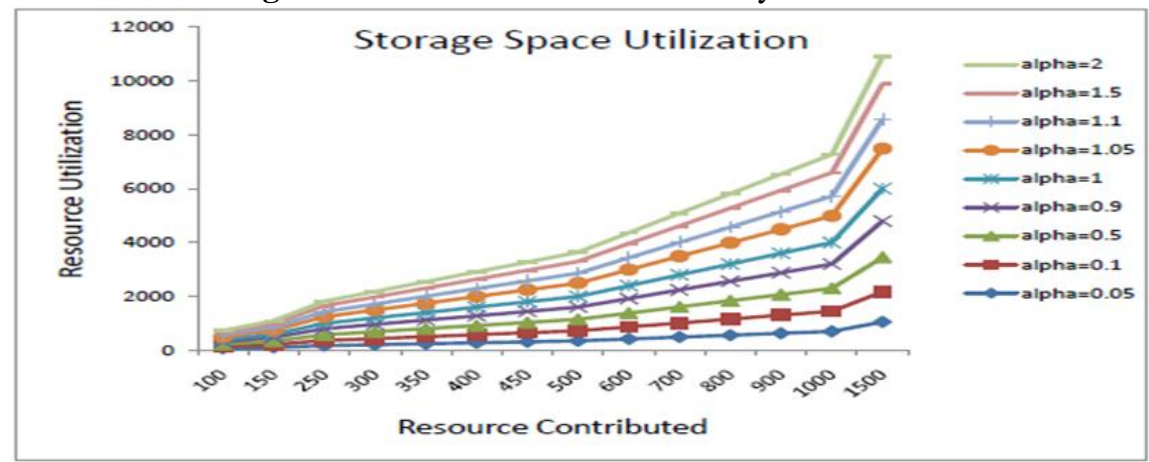

Figure 5 Simulation result of storage space utilization 


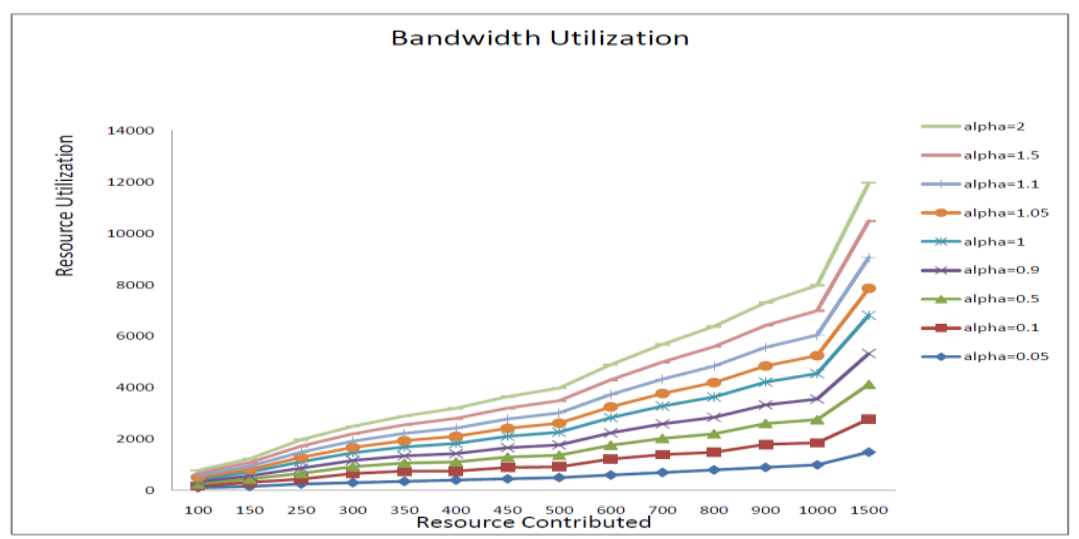

Figure 6: Simulation result of bandwidth utilization

\section{Conclusion AND Future Work}

Grid systems have emerged as an important medium for the exchange of resources. However, more are to be learnt about the impact of users' behaviour on the performance of these systems. This paper is an attempt to analytically model the behaviour of the system and the users. While Grid systems users exhibit characteristics of public and club resources, theyalso differ in various important ways with the aim ofmaximizing profit and performance. Regardless of predictions of the collapse of Grid systems due to freeriding, this drawback can be subduedwhen thesystems arein equilibrium.Overcoming free-riding behaviour is central to the performance and robustness of Grid systems. Many incentive mechanisms have been proposed and implemented with the goal of inducing cooperation from strategic Grid users. The approach presented in this paper also suggests that several important avenues of research on different approaches to free-riding are still possible.In future work, it is being planned to explore how much the performance of these systems could be improved in the absence of free-riding but with other threats, such as Contention, SybilAttack,Collusion, Multiple Identity Attacksetc., in the system. Specifically, it is being planned to extend current work by analyzing thefeasibility and desirability ofnon-price mechanisms for reducing free-riding. Non-price mechanisms may also be important because of the informal nature of the interaction and the difficulty in determining the identity of users.

\section{References}

[1] Abramson, D., Giddy, J. and Kotler, L. (2000): High Performance Parametric Modeling with Nimrod/G: Killer Application for the Global Grid, IPDPS'2000, Mexico, IEEE CS Press, USA, 2000.

[2] Ayodeji Oluwatope, Dauda Iyanda, Ganiyu Aderounmu and Rotimi Adagunodo (2011).Computational Modeling of Collaborative Resources Sharing in Grid System. Information Intelligence, Systems, Technology and ManagementCommunications in Computer and Information Science141 (6):311-321.

[3] Berman, F., and. Fox, G. C. (2003): Grid computing: Making the global infrastructure a reality.

[4] Buchegger, S. and LeBoudec, J.-Y. (2004). A Robust Reputation System for Peer-to-Peer and Mobile Ad-Hoc Networks. In Workshop on Economics of Peer-to-Peer Systems.

[5] Buyya, R.; David Abramson and Jonathan Giddy (2000): Nimrod/G: An Architecture for a Resource Management and Scheduling System in a Global Computational Grid, HPCASIA'2000, China, IEEE CS Press, USA, 2000.

[6] Buyya, R. (2002): Economic-based Distributed Resource Management and Scheduling for Grid Computing. PhD Thesis: Monash University, Melbourne, Australia.

[7] Castro, M., Druschel, P., Ganesh, A., Rowstron, A., and Wallach, D. S. (2002). Security for StructuredPeer-to-Peer Overlay Networks. In Proceedings of Multimedia Computing and Networking2002 (MMCN '02).

[8] Cheng, A. and Friedman, E. (2005). Sybilproof reputation mechanisms. In, ACMSIGCOMM workshop on the Economics of Peerto-Peer Systems (GRIDECON'05).

[9] Chu, Y.-H., Chuang, J., and Zhang, H. (2004). A Case for Taxation in Peer-to-Peer StreamingBroadcast. In Proc. SIGCOMM workshop on Practice and Theory of Incentives and GameTheory in Networked Systems.

[10] Dellarocas, C. (2000). Immunizing Online Reputation Reporting Systems Against Unfair Ratingsand Discriminatory Behavior. In Second ACM Conference on Electronic Commerce.

[11] Dellarocas, C. and Resnick, P. (2003). Online Reputation Mechanisms: A Roadmap for FutureResearch. Summary Report of the First Interdisciplinary Symposium on Online ReputationMechanisms. In Workshop on economics of peer-to-peer networks.ACM SIGecom Exchanges, Vol. 5, No. 4, July 2005.Incentives for Cooperation in Peer-to-Peer Systems _ 49

[12] Douceur, J. R. (2002). The Sybil Attack. In Electronic Proceedings of the International Workshopon Peer-to-Peer Systems.

[13] Feldman, M., Lai, K., Stoica, I., and Chuang, J. (2004a). Robust Incentive Techniques for Peer-to-Peer Networks. In ACM Conference on Electronic Commerce (EC'04).

[14] Feldman, M., Papadimitriou, C., Stoica, I., and Chuang, J. (2004b). Free-Riding and Whitewashingin Peer-to-Peer Systems. In Proc. SIGCOMM workshop on Practice and Theory ofIncentives and Game Theory in Networked Systems.

[15] Foster, I. (2002): What is the Grid? A Three Point Checklist. Daily news and information for the global Grid community 1(6): 55-65

[16] Friedman, E. and Resnick, P. (1998). The Social Cost of Cheap Pseudonyms. Journal of Economics and Management Strategy, 10(2):173 
[17] Gupta, M., Judge, P., and Ammar, M. (2003). A reputation system for peer-to-peer networks. InProceedings of the 13th International Workshop on Network and Operating Systems Supportfor Digital Audio and Video.

[18] Iyanda, D.G., Ogundele, L.A.,Basiru, I.O., and Olatunji, O. (2011).A Prototype for Contention Control in Collaborative Resources Sharing Environment. Journal of Research in Education, Science, Technology and Humanities (JORESTH) 2011, 1(1): 331-345. ISBN: 8129-8-029

[19] Jurca, R. and Faltings, B. (2005). Reputation-based pricing of Grid services. In To appear, ACMSIGCOMM workshop on the Economics of Peer-to-Peer Systems (GRIDECON'05).

[20] Kamvar, S. D., Schlosser, M. T., and Garcia-Molina, H. (2003). The EigenTrust Algorithm forReputation Management in GRID Networks. In Proceedings of the Twelfth International WorldWide Web Conference.

[21] Nabrzyski, J. and J. M. Schopf (2003): Grid Resource Management: State of the Art and Future Trends. Kluwer.

[22] Westerinen, A., Schnizlein, J., Strassner, J., Scherling, M., Quinn, B., Herzog, S, Huynh, A., Carlson, M., Perry, J., and Waldbusser, S. (2001): Terminology for policy based management. IETF RFC 3198, Nov. 2001

[23] Yu, B. and Singh, M. P. (2000). A Social Mechanism of Reputation Management in ElectronicCommunities. In Proceedings of fourth international workshop in cooperative information. 